\title{
Gene influencing in COVID-19 infection, disease severity and its Pharmacotherapy
}

\author{
Sougata Sarkar ${ }^{1}$, Shreyashi Dasgupta ${ }^{1}$, VARTIKA SRIVASTAVA $^{2}$, Satyabrata Sahoo ${ }^{1}$, and \\ Santanu Tripathi ${ }^{1}$ \\ ${ }^{1}$ School of Tropical Medicine \\ ${ }^{2}$ Kalinga Institute of Medical Sciences
}

November 11, 2020

\begin{abstract}
COVID-19 pandemic has badly affected the world, having fatality rate ranging from 1 to $10 \%$ that differs in various countries. The median time from symptoms to clinical recovery is $6-8$ weeks and to death is 2 to 8 weeks. Severity of disease and increasing mortality in COVID 19 is primarily due to presence of comorbidities like cardiovascular disease, pre-existing lungs disease, hypertension, diabetes, obesity and cancer. It is already known to us that humans show difference in drug responses because of their varied genetic make-up. Population genomics furnish an insight about genetic characteristic of a populations and it is critical in determining susceptibility, severity and natural protection against infectious diseases. Therefore, understanding the population genetic makeup may be deemed necessary to identify those who are at risk or protective from disease and develop genomics information, that would be useful in providing insight about COVID-19 disease severity or outcomes. Some of the proposed genetic gateways in COVID 19 pathogenesis are mentioned in this review including roles of ACE2 gene, HLA gene, Chromosome 3P21.31, ABO locus, genes responsible for cytokine storm, TLR-pathway, Family Mediterranean fever and G6PD deficiency. Significant interindividual variability in response to drug therapy exists in patients. This review also evaluates the current therapeutics in COVID-19 like hydroxycholoroquine, azithromycin, RNA polymerase inhibitors, interleukin inhibitors, antivirals, ivermectin, doxycyclin and their pharmacogenomics viewpoint. Such Pharmacogenomic studies are very helpful for the physicians to choose and give accurate first line therapy for COVID 19 patients.
\end{abstract}

\section{Hosted file}

gene $\mathrm{n}$ covid ready.pdf available at https://authorea.com/users/374730/articles/492189-geneinfluencing-in-covid-19-infection-disease-severity-and-its-pharmacotherapy 